cosmic ray electron spectrum implies that an increase from $0.5 \mathrm{eV} \mathrm{cm}^{-3}$ in the energy density of the microwave radiation (which will oceur, for example, with a spectrum of the form $I(\lambda) \propto \lambda-x$ extending below a wavelength $\lambda=1.7 \mathrm{~mm}$ ) will result in severe difficulties in our understanding of the propagation and the life-time of electrons.

It is evident from the upper limits in Fig. 1 that the microwave spectrum which is approximately of the form $I(\lambda) \propto ;-x$ in the experimentally measured region cannot be of the same form below $2 \mathrm{~mm}$. The results in Fig. 1 are consistent with a blackbody spectrum with a temperature of $3^{\circ} \mathrm{K}$ and indeed show that, if the radiation is a blackbody radiation, the temperature cannot exceed $3^{\circ} \mathrm{K}$. The suggestion of Narlikar and Wickramasinghe ${ }^{6}$ that the spectrum is approximately of the form $I(\lambda) \propto \lambda^{-x}$, with a cut-off, is not inconsistent with the results in Fig. 1, but perhaps requires special hypotheses concerning "dust grains" to explain all of the spectrum in Fig. 1. Further refinement of the cosmic ray data, especially the gammaray observations on the Crab nebula, is expected in the near future and should improve the limits on microwave intensities in the universe.

This investigation arose from a question put by $\mathrm{Dr}$ M. S. Vardya of Tata Institute, Bombay, to whom I am grateful for several discussions. Thanks are due to $\mathrm{Dr}$ G. G. Fazio for some comments. This work was done while I held a National Research Council senior postdoctoral visiting research associateship supported by the Smithsonian Institution.

M. V. Krishna Apparao*

Smithsonian Astrophysical Observatory,

Cambridge,

Massachusetts.

Received July $18,1968$.

* On leave from the Tata Institute of Fundamental Research, Bombay, India.

1 Penzias, A. A., and Wilson, R. W., Astrophys. J., 142, 419 (1965).

${ }^{2}$ Gamow, G., in Vistas in Astronomy (edit. by Beer, A.), 2, 1726 (Pergamon Press, New York, 1956).

${ }^{3}$ Dicke, R. H., Peebles, P. J. F., Roll, P. G., and Wilkinson, D. T., Astrophys. $J ., 142,414$ (1965).

4 Shakeshaft, J. R., and Webster, A. S., Nature, 217, 339 (1968).

${ }^{5}$ Hoyle, F., and Wickramasinghe, N. C., Nature, 214, 969 (1967).

- Narlikar, J. V., and Wickramasinghe, N. C., Nature, 216, 43 (1967).

i Apparao, M. V. K., Proc. Ind. Acad. Sci., 65A, 349 (1967).

${ }^{8}$ Fazio, G. G., Ann. Rev. Astron. Astrophys., 5, 481 (1967).

${ }^{\circ}$ Fazio, G. G., Helmken, H. F., Cavrak, jun., S. J., and Hearn, D. R., Canad. $J$. Phys. (in the press).

\section{Radial Velocity Measurements on the Zodiacal Light Spectrum}

THFre has been speculation for some time about the possibility of detecting the motion of zodiacal dust particles by accurately measuring the wavelength position of a solar Fraunhofer absorption line scattered in the cloud. Ingham ${ }^{1}$ derived the $H \beta$ absorption line profile that would result from solar radiation scattered by dust particles in circular Keplerian orbjts about the Sun. He showed that, in the ecliptic, at an elongation of $30^{\circ}$ from the Sun, the expected wavelength shift is $0.3 \AA$ to the bluo for dust particles co-rotating with the Earth, and $0.8 \AA$ to the red for contra-rotating dust. Ring et al. ${ }^{2}$ made measurements on the $\mathrm{H} \beta$ absorption line from Mount Chacaltaya, in the Bolivian Andes, and found a blue wavelength shift in the evening zodiacal light, a possible interpretation of which could be in terms of a zodiacal dust cloud corotating with the Earth at twice the expected circular orbital velocity. During a programme of observations to map the night sky $\mathrm{H} \beta$ emission line ${ }^{3}$, many spectra were recorded in the region of the evening zodiacal light between

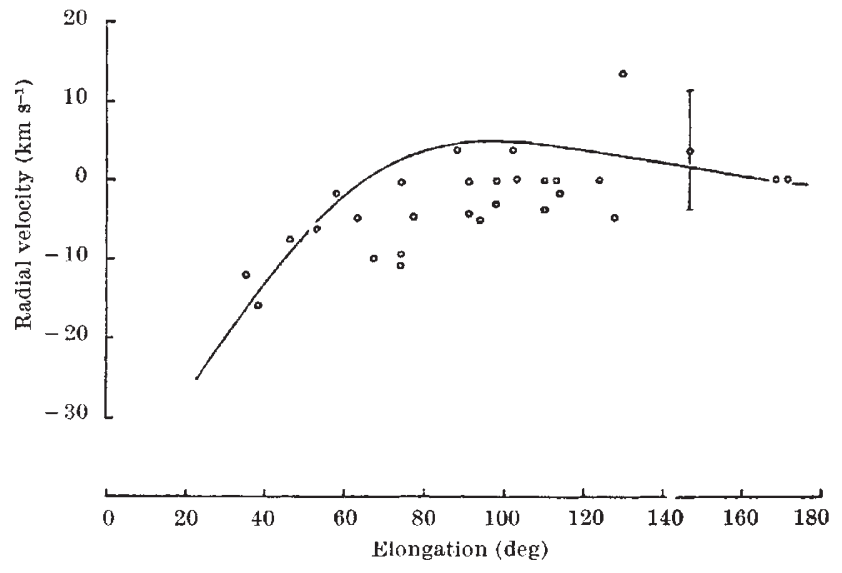

Fig. 1. Variation of radial velocity with elongation in the ecliptic for the evening zodiacal light. The theoretical variation assuming circular Keplerian orbits and an $r^{-3}$ spatial dersity distribution is represented

elongations of $35^{\circ}$ and $180^{\circ}$ from the Sun. The observations were made with a gas pressure scanned Fabry-Perot interferometer ${ }^{4}$, working at a resolving power of 8,000 , from an 11,500 foot observatory on Testa Grigia in the Italian Alps (lat. $45^{\circ}$ N.). The instrument scanned, in steps of $0 \cdot 14 \AA$, a range of $2 \AA$ centred on the $4861 \cdot 33 \AA$ laboratory $H \beta$ wavelength, thus providing about 14 closely spaced points across the spectral feature. Wavelength calibrations were made before and after each spectrum was recorded.

Although all spectra showed an emission feature at the $H \beta$ wavelength, it was found to be relatively easy to interpolate the background zodiacal light absorption line. After subtraction of the emission feature the absorption line could be positioned in wavelength to an accuracy of $\pm 0.14 \AA$, that is, $\sim \pm 8 \mathrm{~km} \mathrm{~s}^{-1}$ in radial velocity. The results of the analysis are shown in Fig. 1 for the evening zodiacal light, the negative radial velocity corresponding to a blue wavelength shift of the absorption line and the positive radial velocity to a red shift. Also shown in the figure, for comparison, is the radial velocity variation that would be expected of dust particles in circular Keplerian orbits about the Sun, in the same sense as the Earth, and with a spatial density distribution falling as $r^{-3}$ from the Sun. The agreement is good for elongations up to $60^{\circ}$; above this value, theory and experiment deviate.

Unfortunately, no corresponding spectra were obtained in the morning zodiacal light. A red shift at small elongations in the morning, considered with the evening blue shift, would strongly favour the hypothesis that the zodiacal dust is in orbit about the Sun and co-rotaling with the Earth. A morning blue shift might indicate that the dust is falling into the Sun'.

Further observations are planned this coming winter, when the radial velocity variation with both elongation and ecliptic latitude will be investigated. For this purpose an absorption line thought to be free from emission, probably the $5183 \AA$ magnesium line, will be used.

\section{N. K. REAY \\ J. RING}

Imperial College of Science and Technology, London.

Received July 18, 1968

${ }^{1}$ Ingham, M. F., Mon. Not. Roy. Astro. Soc., 126, 377 (1963),

${ }^{2}$ Ring, J., Clarke, D., James, J. F., Daehler, M., and Mack, J. E., Nature,202, 167 (1964).

${ }^{a}$ Hindle, P. H., Reay, N. K., and Ring, J., Planet. Spa. Sci., 16, 795 (1968).

- Hindle, P. H., and Reay, N. K., J. Sci. Inst., 44, 360 (1967). 